\title{
COMT genotype influences prefrontal response to emotional distraction
}

\author{
SONIA J. BISHOP \\ University of Cambridge, Cambridge, England \\ and MRC Cognition \& Brain Sciences Unit, Cambridge, England \\ JONATHAN D. COHEN \\ Princeton University, Princeton, New Jersey \\ JOHN FOSSELLA and B. J. CASEY \\ Weill Medical College of Cornell University, New York, New York \\ and \\ MARTHA J. FARAH \\ University of Pennsylvania, Philadelphia, Pennsylvania
}

\begin{abstract}
Early studies of genetic effects on brain activity have been conducted to investigate primarily either the influence of polymorphisms in dopaminergic genes, especially the catechol- $O$-methyltransferase (COMT) gene, on prefrontal cognitive processes such as working memory, or that of polymorphisms in the serotonin transporter gene on the amygdala response to threatening stimuli. Here, we address genetic influences on the neural systems underlying cognitive-affective interactions. Specifically, we assess the effect of the COMT val ${ }^{158}$ met polymorphism on frontal regulation of attention under emotional distraction. Healthy volunteers were scanned while performing a house-matching task with affectively negative versus neutral distractors. Effects of val allele load were examined on frontal regions associated with attentional control and emotion regulation, and on parahippocampal regions associated with perception of houses. As we predicted, val load correlated positively with activity in control- and task-related regions during performance under emotional distraction. These findings provide an initial step toward identifying genetic contributions to interindividual variability in recruitment of mechanisms that regulate affective processing.
\end{abstract}

One of the most exciting recent developments in functional neuroimaging is the discovery that fMRI and other imaging methods are capable of revealing the neural correlates of individual differences in psychological processing among healthy, normal people. In contrast to earlier work, in which the activity of typical or average brains (Posner \& Raichle, 1995) or differences between normal and pathological brains (Frith \& Dolan, 1998) were mapped, in recent studies correlations have been made between differences in brain activity during cognitive or affective processing and normal individual variation

This study was supported by the Center for Cognitive Neuroscience at the University of Pennsylvania and the Center for the Study of Brain, Mind, and Behavior at Princeton University. Funding was provided by the Institute for Research in Cognitive Science, University of Pennsylvania, and by NIH Grants R21-DA01586, R01-HD043078, R01-DA14129, R01-DA18913, and P30-HD269-04S2. DNA extraction and genotyping were conducted at the Weill Medical College of Cornell University. Our thanks go to Geoff Aguirre for advice on imaging analysis and to Kathleen Durkin and Andrew Engell for assistance with fMRI data collection. Correspondence should be addressed to S. J. Bishop, MRC/Wellcome Trust Behavioural and Clinical Neuroscience Institute, Department of Experimental Psychology, University of Cambridge, Downing Street, Cambridge CB2 3EB, England (e-mail: sb445@cam.ac.uk). in characteristics such as intelligence (Gray, Chabris, \& Braver, 2003), anxiety (Bishop, Duncan, Brett, \& Lawrence, 2004; Etkin et al., 2004), extraversion, and neuroticism (Canli, Silvers, Whitfield, Gotlib, \& Gabrieli, 2002; Canli et al., 2001).

Neuroimaging is also being used to investigate how specific genetic polymorphisms influence individual differences in neurocognitive function. This approach is particularly valuable when polymorphisms are identified that are common (i.e., those that possess two or more different alleles that are fairly frequent in the general population) and have functional consequences for brain activity - for example, by altering neurotransmitter metabolism.

A compelling case has been made for the use of a polygenic framework in considering the contribution of such genetic factors to common psychiatric conditions, given the mixed findings of many association studies (for a review, see Comings, 2001). However, it has been argued that genetic influences may be observed more clearly at the neurophysiological level, as measured by the bloodoxygenation-level-dependent (BOLD) signal in fMRI, than at the level of clinical diagnosis or behavior, making it viable to detect small changes in variance accounted for by single polymorphisms (Goldberg \& Weinberger, 
2004). Indeed, polygenic contributions to psychiatric disorders may reflect a combination of genetic influences on different neurocognitive mechanisms. These influences may also contribute to variability in cognitive and affective profiles within the population as a whole. A genetic imaging approach allows us to focus on specific neurocognitive mechanisms and to examine how particular genetic polymorphisms account for variability between individuals in neural recruitment. As initial small-scale studies such as the one described here sketch out a picture of polymorphisms of potential interest, larger scale studies can build upon them to examine gene $\times$ gene interactions and dissociations.

In the present study, we used a genetic imaging approach to investigate whether or not the catechol- $O$ methyltransferase (COMT) val ${ }^{158}$ met polymorphism influences frontal cortical activation during task conditions requiring regulation of attention to threat-related distractors. This is an initial attempt to examine the influence of single genetic polymorphisms on activation of the neural mechanisms governing cognitive-affective interactions.

COMT degrades both dopamine and norepinephrine. The polymorphism of interest involves the substitution of methionine for valine at codon 158 (Lachman et al., 1996). The enzyme containing met ${ }^{158}$ is unstable at $37^{\circ} \mathrm{C}$ and has roughly one quarter of the activity of the val ${ }^{158}$ enzyme (Lotta et al., 1995). The alleles are codominant, with heterozygous individuals having levels of enzyme activity that are midway between those of homozygous individuals (Weinshilboum, Otterness, \& Szumlanski, 1999). Given the scarcity of dopamine transporter in the prefrontal cortex (PFC; Lewis, Sesack, Levey, \& Rosenberg, 1998; Sesack, Hawrylak, Matus, Guido, \& Levey, 1998), the COMT val ${ }^{158}$ met polymorphism has been suggested to have an especially critical influence on dopamine levels in this region. Specifically, possession of the more active val allele is thought to be associated with increased metabolism and, consequently, lower levels of synaptic dopamine (Mattay et al., 2003; but see Bilder, Volavka, Lachman, \& Grace, 2004, for an account that distinguishes between potentially opposing effects of the COMT val ${ }^{158}$ met polymorphism on phasic and tonic dopamine mechanisms).

Prefrontal function is optimized within a relatively narrow range of dopaminergic activity (Arnsten, 1998; Goldman-Rakic, 1998). Hence, differences in the metabolism of prefrontal dopamine associated with the COMT val ${ }^{158}$ met polymorphism may impact measurably on prefrontal function. In line with this view, a number of studies have reported an effect of COMT genotype on behavioral performance and/or neural activity during tasks that tap PFC function. These studies have focused primarily on purely "cognitive" processing - in particular, working memory and set shifting. Val allele load has been found to be positively correlated with PFC activation during performance of "two-back" tests of working memory (Egan et al., 2001) and negatively correlated with behavioral measures of performance - accuracy in particular - both for two-back tests (as reported within a separate second study - Goldberg et al., 2003) and for the Wisconsin card sorting task (Bruder et al., 2005; Egan et al., 2001; Malhotra et al., 2002). However, in other tests of prefrontal function, including reversal learning and regulation of response competition, val allele load has been positively associated with performance accuracy (Fossella et al., 2002; Nolan, Bilder, Lachman, \& Volavka, 2004), resulting in an ongoing debate relating to the factors determining the association observed between COMT genotype and task performance (Bilder et al., 2004).

In contrast to this body of work, the initial genetic imaging studies of affective processing have focused primarily on a polymorphism in the serotonin transporter gene (5HTT-LPR) and the extent to which it modulates amygdala function. Possession of one or two copies of the short allele is associated with reduced serotonin reuptake and an augmented amygdala response to negatively valenced stimuli (Hariri et al., 2005; Hariri et al., 2002; see Brown \& Hariri, 2006). This research focus is not surprising given the large literature on the role of the amygdala in the response to emotional stimuli. However, cognitive and affective systems are not orthogonal, and recently we and others have found that regions implicated in cognitive control may also play a crucial role in the regulation of the processing of emotional stimuli and the experience of affective states (Bishop, Duncan, Brett, \& Lawrence, 2004; Casey, 2005; Ochsner \& Gross, 2005). In one example, we built on the extensive literature demonstrating a role of the PFC in controlling attention to distractor stimuli (Casey, 2005; Miller \& Cohen, 2001) by investigating top-down influences on the processing of task-irrelevant negative stimuli (Bishop, Duncan, Brett, \& Lawrence, 2004). We showed increased prefrontal recruitment in response to heightened expectancy of threat-related distractors, the magnitude of the response observed being reduced in individuals reporting high levels of state anxiety.

These findings led us to question whether genetic influences on frontal cortical function might affect the regulation of emotional processing. In addition to reports of a positive association between COMT val allele load and PFC activation during cognitive performance, there is some evidence to suggest that the COMT met allele may be associated with increased levels of anxiety-related symptomatology (Enoch, Xu, Ferro, Harris, \& Goldman, 2003; Karayiorgou et al., 1997; Olsson et al., 2005; Woo et al., 2004 ) and with reduced ability to regulate the response to negative stimuli such as pain (Zubieta et al., 2003). Given these findings, we were interested in investigating the impact of the COMT val ${ }^{158}$ met polymorphism on frontal regulation of attentional control over negatively valenced stimuli. Our specific prediction was that the number of met alleles possessed ( $0-2$ ) would be negatively correlated with the recruitment of frontal cortical regions in response to negative distractor stimuli, paralleling the relationship with anxiety reported by Bishop, Duncan, Brett, and Lawrence (2004). Since both lateral and orbital regions of the 
frontal cortex have been implicated in controlled processing in different studies (see Casey, Tottenham, \& Fossella, 2002, and Ochsner \& Gross, 2005, for reviews), we investigated the influence of COMT genotype in both lateral $\mathrm{PFC}$ and orbitofrontal regions of interest (ROIs).

\section{METHOD}

\section{Participants}

Thirteen participants ( 6 female and 7 male, all right-handed, 18-30 years of age) performed an adapted version of the matching task used by Vuilleumier, Armony, Driver, and Dolan (2001) and by Bishop, Duncan, Brett, and Lawrence (2004; Bishop, Duncan, \& Lawrence, 2004) while fMRI data were collected. The study was approved by Princeton University's Internal Review Panel and was performed in compliance with its guidelines. Individuals with a history of inpatient psychiatric care, neurological disease, or head injury were excluded, as were individuals on medication for anxiety or depression. In addition, the Princeton standard exclusion criteria for fMRI studies (e.g., metal free, absence of pacemakers) were followed.

\section{Task}

Visual stimuli were projected onto a screen at the rear of the bore of the magnet. The participants viewed these images via an angled mirror attached to the RF head coil and placed above their eyes. On each trial, the participants were shown a brief visual display comprising two pictures taken from the International Affective Picture System (IAPS; Lang, Ohman, \& Vaitl, 1988) and two pictures of houses presented in vertical and horizontal pairs around a central fixation cross (see Figure 1).

There were two imaging acquisition runs, each consisting of 16 blocks of three trials with a within-blocks mean interstimulus inter- val of $5 \mathrm{sec}$. At the start of each block, a cue indicated whether the participants should attend to the vertical or the horizontal pair of pictures. The houses were always presented in the attended positions, and the participants had to indicate via a keypress whether or not they were identical. The IAPS pictures were task-irrelevant distractors. ${ }^{1}$ Trials were split into negative distractor and neutral distractor blocks. The negative distractor blocks comprised trials in which the distractor pictures were negatively valenced. The neutral distractor blocks comprised trials in which the distractor pictures were neutral or mildly positively valenced. The mean valence and arousal ratings of the pictures in the negative and neutral distractor blocks were 2.3 $(S E=.11)$ and $5.3(S E=.09)$, respectively, for valence and $6.1(S E=$ $.11)$ and $3.2(S E=.11)$, respectively, for arousal. Both ratings were made on a nine-point scale, with scores from 1 to 9 representing increasing positivity (valence scale) and arousal (arousal scale). This matching task was closely based on the paradigm used by Bishop, Duncan, Brett, and Lawrence (2004), focusing on those conditions in which frontal activation was observed: short strings of all negative distractor trials versus short strings of all neutral distractor trials.

\section{Image Acquisition}

BOLD contrast functional images were acquired with echoplanar T2*-weighted EPI imaging using a 3T Siemens Allegra scanner. Each image volume consisted of 31 interleaved 5-mm-thick axial oblique slices, providing whole-brain coverage with an in-plane resolution of $3 \times 3 \mathrm{~mm}$ (repetition time $=3,000 \mathrm{msec}$, echo time $=$ $27 \mathrm{msec}$, flip angle $=90^{\circ}$ ). Data were acquired in two scanning runs of approximately $5 \mathrm{~min}$ each. The first nine volumes of each run were discarded to allow for T1 equilibration effects.

\section{Image Analysis}

Data were analyzed using SPM 99 software (Wellcome Department of Imaging Neuroscience, London). Standard preprocessing

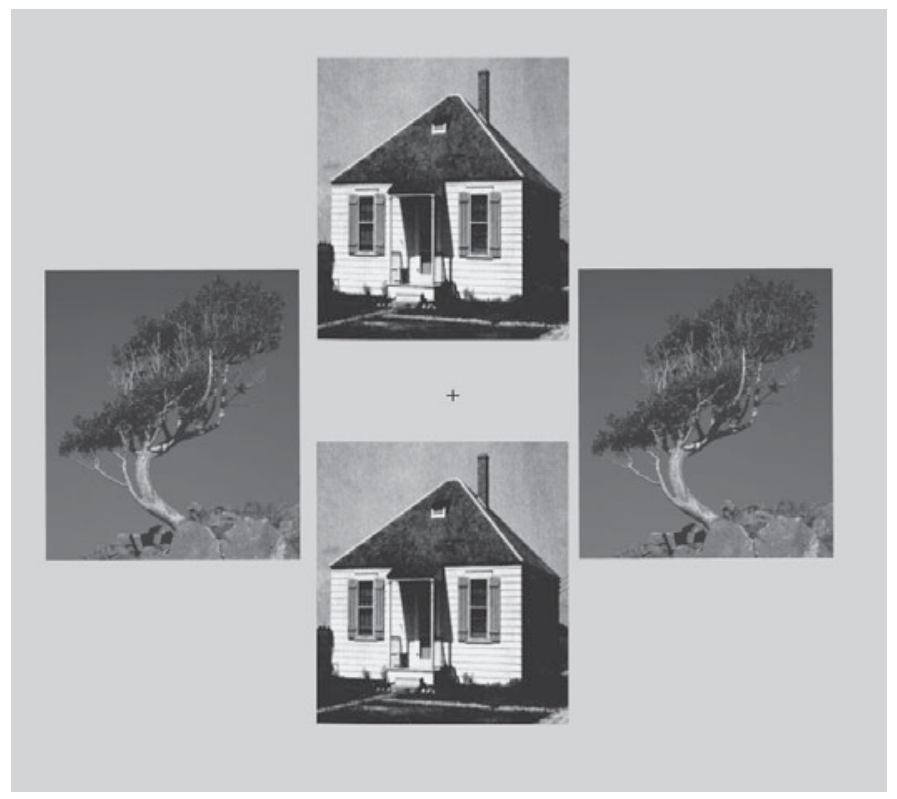

Figure 1. Example stimuli. On each trial, two pictures of houses and two pictures from the International Affective Picture System (Lang et al., 1988) were presented in vertical and horizontal pairs around a central fixation cross. (Here, for copyright reasons, we have replaced the IAPS pictures in the display with similar pictures from another source.) The participants attended to the houses after being cued spatially as to their locations for each block of trials and decided whether they were identical $(p=.05)$ or not. The unattended pictures were identical and either negative or neutral in valence. 
was conducted, which comprised slice timing correction, realignment, and normalization of each participant's EPI data to the Montreal Neurological Institute's MNI/ICBM template. Images were resampled into this space with 2-mm isotropic voxels and smoothed with a Gaussian kernel of $8 \mathrm{~mm}$ full width at half maximum. Trials were modeled with step functions $.25 \mathrm{sec}$ in duration, convolved with the canonical hemodynamic response function to form regressors. Temporal derivatives of these regressors were also included, as were realignment parameters for each session, in order to account for residual movement-related variance. A 120 -sec high-pass filter was used to remove low-frequency noise. A random effects analysis was used to analyze data at a group level, and modulations by COMT val ${ }^{158}$ met genotype were assessed by simple regression against val allele load $(0-2$, where $0=$ met met, $1=$ met val, and $2=$ val val $)$.

Bilateral ROIs were created for dorsolateral PFC (DLPFC), ventrolateral PFC (VLPFC), and orbitofrontal cortex (OFC). The DLPFC and VLPFC ROIs were taken from Bishop, Duncan, Brett, and Lawrence (2004) and comprised 8-mm-radius spheres with the following central coordinates: DLPFC, $\pm 34,36,24$; VLPFC, \pm 38 , 20, 0. Following previous reviews (Duncan \& Owen, 2000; Owen, 1997), the DLPFC region included parts of the middle frontal gyrus and inferior frontal sulcus, whereas the VLPFC region included parts of the frontal operculum and anterior insula. Given the problems with signal dropout and associated difficulties in normalization in OFC, slightly larger (10-mm-radius) spheres were used for the OFC ROIs. ${ }^{2}$ Here, the central coordinates $( \pm 24,34,-12)$ were taken from activations reported by Wager et al. (2004) in response to expectancy of negative stimuli (pain) under placebo conditions, which were held to reflect regulation of affective processing.

In addition to these frontal ROIs, bilateral ROIs were created for parahippocampal cortex and the amygdala. Pictures of houses have been shown to produce a strong bilateral response in a region of parahippocampal cortex now known as the parahippocampal place area (PPA; Epstein \& Kanwisher, 1998). In the present study, bilateral PPA ROIs were created using 8 -mm spheres centered on coordinates $( \pm 28,-44,-14)$ obtained by averaging PPA activation peaks reported for attending to houses versus attending to faces in the studies from which the current matching paradigm was adapted (Bishop, Duncan, \& Lawrence, 2004; Vuilleumier et al., 2001). Finally, for the amygdala ROIs, we used bilateral anatomical ROIs from the Montreal Neurological Institute (Tzourio-Mazoyer et al., 2002). Voxel-wise comparisons were conducted and small-volume corrections applied for activations within each ROI (Worsley et al., 1996). In addition, an ROI-based correlational analysis was conducted at the random effects level. The MarsBaR ROI toolbox (Brett, Anton, Valabregue, \& Poline, 2002) was used to extract and average across the activation for all voxels within a given ROI. This enabled us to examine how activity in different ROIs covaried across participants and whether this differed by trial type. All activations are reported using MNI coordinates.

\section{Genotyping}

All the volunteers gave informed consent for a buccal swab to be obtained using a buccal brush. DNA was isolated using the MasterAMP Buccal Swab and DNA Extraction Kit (Epicentre Technologies, Madison, WI). This provides yields of $0.5-3 \mu \mathrm{g}$ of DNA from each buccal sample. Taq polymerase, PCR buffer, and dNTPs were obtained from QIAGEN and used at concentrations recommended for a $20-\mu 1$ PCR reaction. A "touchdown" PCR cycling regimen and the addition of DMSO ( $10 \%$ final v:v) were used in order to optimize the hybridization stringency. Forward 5-ACTGTGGCTACTCAGCTGTG-3 and reverse 5-CCTTTTTCCAGGTCTGACAA-3 primers were used following Daniels et al. (1996). PCR conditions were as follows: $94^{\circ} \mathrm{C}$ for 3-min initial heating, followed by 12 cycles of $94^{\circ} \mathrm{C}$ for $30 \mathrm{sec}$, $58^{\circ} \mathrm{C}$ for $45 \mathrm{sec}$, and $72^{\circ} \mathrm{C}$ for $30 \mathrm{sec}$; and finally, 28 cycles of $94^{\circ} \mathrm{C}$ for $30 \mathrm{sec}, 50^{\circ} \mathrm{C}$ for $45 \mathrm{sec}$, and $72^{\circ} \mathrm{C}$ for $30 \mathrm{sec}$. This process was followed by restriction digestion with NlaIII. Gel electrophoresis in MetaPhor agarose followed by staining in ethidium bromide was used to resolve and visualize DNA fragments.

\section{RESULTS}

\section{Imaging Analyses}

Bishop, Duncan, Brett, and Lawrence (2004) showed an inverse correlation between participants' self-reported anxiety levels and frontal cortical activation to strings of house-matching trials with negative versus neutral distractors. In the present study, we investigated whether there would be a similar inverse correlation with met allele load and, hence, a positive correlation with val allele load (i.e., individuals with two val alleles and no met alleles showing greatest frontal activation; individuals with two met alleles and no val alleles showing least frontal activation; and heterozygotes, or individuals with one val allele and one met allele, showing intermediate levels of activation). The results of our simple regression analysis at the random effects level showed that val allele load was positively associated with the magnitude of both the left $\operatorname{VLPFC}(x, y$, $z=-32,18,2 ; Z=3.28, p<.05$ small volume corrected [svc] $)$ and the left $\operatorname{OFC}(x, y, z=-22,26,-16 ; Z=4.16$, $p<.01 \mathrm{svc}$ ) response to negative distractor versus neutral distractor trials (Figures 2A and 2B). There was no significant relationship between val allele load and activation in any of the other frontal ROIs $(p>.1, \mathrm{svc})$.

If the frontal cortical response to negative versus neutral distractor trials reflects the recruitment of control mechanisms to facilitate task-related processing, one might expect activation in structures associated with the processing of task-related stimuli (houses) to show similar modulation by COMT genotype. In line with this, val allele load was positively associated with the magnitude of the PPA response to negative versus neutral distractor trials within both the left $(x, y, z=-28,-36,-14 ; Z=$ $4.01, p<.01 \mathrm{svc})$ and right $(x, y, z=30,-40,-20 ; Z=$ $3.57, p<.02 \mathrm{svc}$ ) ROIs (see Figure $2 \mathrm{C}$ ). ${ }^{3}$

We also examined whether or not the amygdala response to negative versus neutral distractor trials was modulated by COMT genotype. Here, no significant relationship between val allele load and left or right amygdala response to negative versus neutral distractor trials was detected $(p \mathrm{~s}>.1 \mathrm{svc})$.

An ROI-based correlational analysis (see the Method section) revealed that, for negative distractor trials, left OFC activity was significantly correlated with left amygdala activity $(r=.57, p<.05)$ but not with PPA activity $(p \mathrm{~s}>.1)$, whereas activity in both VLPFC ROIs was correlated with activity in both PPA ROIs (left VLPFC, left PPA: $r=.66, p<.02$; left VLPFC, right PPA: $r=.59, p<$ .05 ; right VLPFC, left PPA: $r=.65, p<.02$; right VLPFC, right PPA: $r=.60, p<.05)$. The strongest correlation was between left VLPFC activity and left OFC activity $(r=.76$, $p<.005)$. Left VLPFC and left OFC activity were also correlated during neutral distractor trials $(r=.70, p<.01)$, as 
A

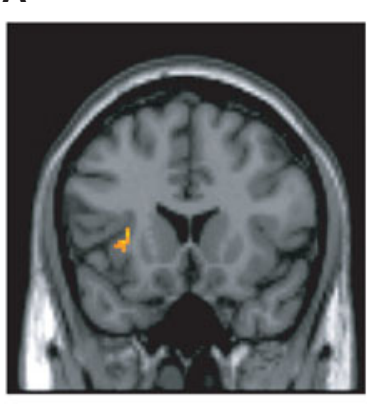

$y=18$

B

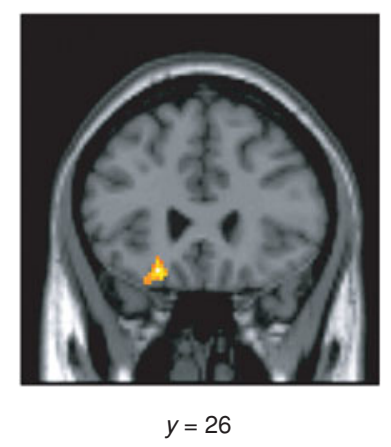

C

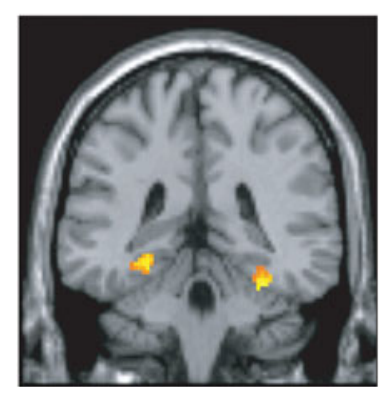

$y=-38$
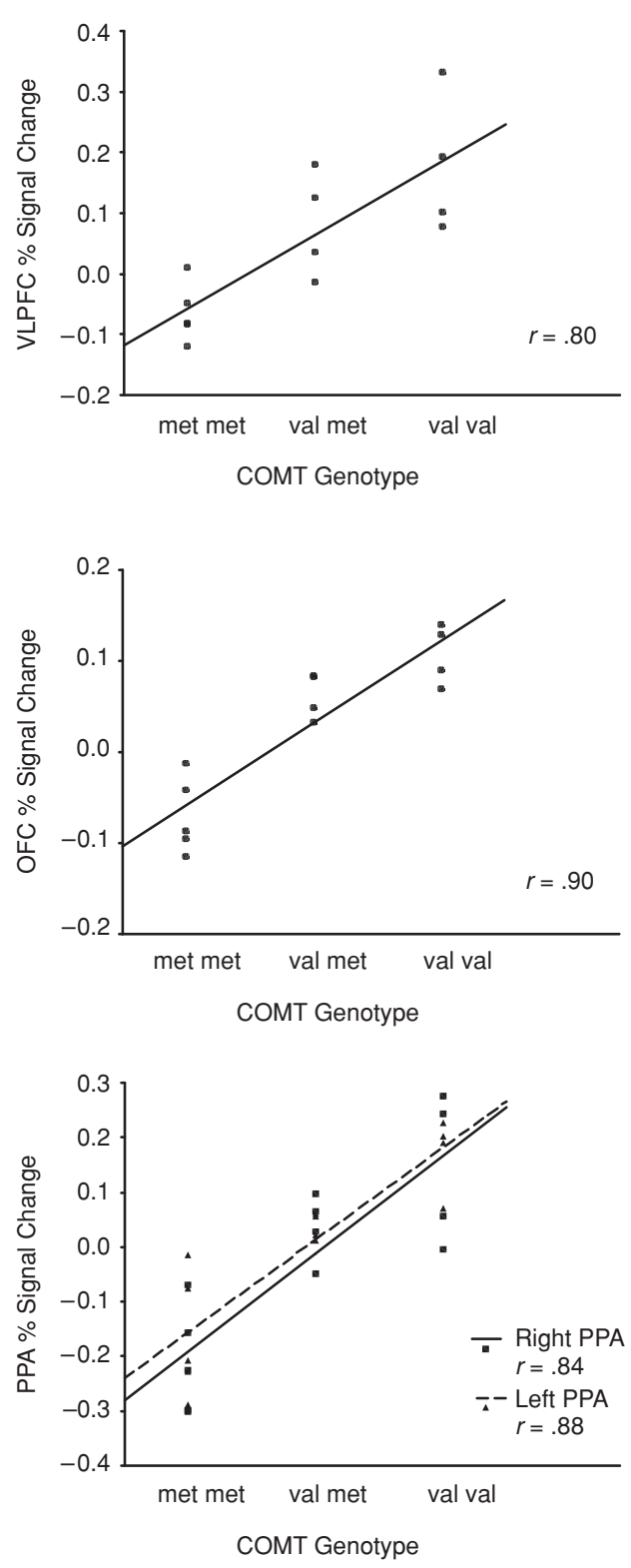

Figure 2. Correlation between COMT val ${ }^{158}$ allele dosage (0-2) and activation to negative versus neutral distractor trials for $(A)$ left ventrolateral prefrontal cortex (VLPFC), (B) left orbitofrontal cortex (OFC), and (C) bilateral parahippocampal place area (PPA). Left panels show statistical maps (thresholded at $t=3.6$, equivalent to $p<.1$ corrected for 8-mm spherical regions of interest (ROIs) and $p<.002$ whole brain uncorrected, with a minimum spatial extent of two voxels). Activations extending outside the ROIs are included where contiguous with the cluster within the ROI up to a maximum radius of $12 \mathrm{~mm}$ from the ROI center. Right panels display neural responses (mean percent signal change for negative versus neutral distractor trials) against COMT genotype. Individual BOLD responses were extracted from the peak voxel within each ROI shown to the left. Coordinates as given in the main text.

were activity between each of these regions and right PPA activity $(r=.77, p<.005$ and $r=.71, p<.01$, respectively). There was no relationship between amygdala activity and OFC activity for these trials $(p s>1)$.

\section{Behavioral Results and Brain-Behavior Correlations}

Mean reaction times for negative and neutral distractor trials are given by COMT genotype in Table 1 . In ad- 
Table 1

Behavioral Data: Reaction Times (RTs, in Milliseconds) and Error Rates (\%Error) for the House-Matching Task by Distractor Valence and COMT Genotype

\begin{tabular}{|c|c|c|c|c|c|c|c|c|}
\hline \multirow[b]{3}{*}{ COMT Genotype } & \multicolumn{4}{|c|}{ RT } & \multicolumn{4}{|c|}{$\%$ Error } \\
\hline & \multicolumn{2}{|c|}{$\begin{array}{c}\text { Negative } \\
\text { Distractor } \\
\text { Trials } \\
\end{array}$} & \multicolumn{2}{|c|}{$\begin{array}{c}\text { Neutral } \\
\text { Distractor } \\
\text { Trials } \\
\end{array}$} & \multicolumn{2}{|c|}{$\begin{array}{c}\text { Negative } \\
\text { Distractor } \\
\text { Trials } \\
\end{array}$} & \multicolumn{2}{|c|}{$\begin{array}{c}\text { Neutral } \\
\text { Distractor } \\
\text { Trials }\end{array}$} \\
\hline & $M$ & $S E$ & $M$ & $S E$ & $M$ & $S E$ & $M$ & $S E$ \\
\hline \multicolumn{9}{|l|}{ Sample 1} \\
\hline All genotypes $(n=13)$ & 818.0 & 38.3 & 817.4 & 41.6 & 13.5 & 2.4 & 12.7 & 2.8 \\
\hline val val $(n=4)$ & 805.9 & 69.0 & 814.1 & 70.7 & 11.3 & 5.3 & 11.3 & 8.1 \\
\hline val met $(n=4)$ & 869.7 & 90.8 & 867.4 & 95.2 & 15.0 & 4.6 & 13.5 & 3.7 \\
\hline met met $(n=5)$ & 786.3 & 53.1 & 779.9 & 64.9 & 14.2 & 3.5 & 13.2 & 3.4 \\
\hline \multicolumn{9}{|l|}{ Combined sample } \\
\hline All genotypes $(n=53)$ & 806.6 & 20.3 & 810.8 & 20.5 & 9.5 & 0.9 & 9.7 & 1.0 \\
\hline val val $(n=16)$ & 816.3 & 30.6 & 828.2 & 29.7 & 8.6 & 1.7 & 8.9 & 1.5 \\
\hline val met $(n=20)$ & 816.0 & 33.5 & 809.3 & 31.9 & 9.9 & 1.5 & 10.9 & 1.5 \\
\hline met met $(n=17)$ & 786.5 & 41.5 & 796.2 & 45.1 & 9.8 & 1.6 & 9.2 & 1.7 \\
\hline
\end{tabular}

Note-Data are presented both for the participants in the fMRI study reported here (Sample 1) and for a larger combined sample including genotyped volunteers who completed the house-matching task without acquisition of fMRI data.

dition to the behavioral data for the study reported here (Sample 1), we present the behavioral data for a larger combined sample that included an additional 40 volunteers genotyped for the COMT val ${ }^{158}$ met polymorphism who completed the house-matching task without fMRI data being collected. For both samples, there was no significant effect of distractor valence or of COMT genotype and no significant interaction of distractor valence $\times$ COMT genotype $(p s>.1)$. This was true for both the reaction time and the accuracy data. There was, however, a trend for increased PPA activity for negative versus neutral distractor house-matching trials to be associated with reduced reaction times for negative versus neutral distractor trials $\left(r=-.37, p=.1\right.$, one-tailed). ${ }^{4}$ In other words, increased activity in this "task-related processing" region was marginally associated with reduced RT costs from negative distractors. Neither of the other regions where activity to negative versus neutral distractors was modulated by COMT genotype (VLPFC and OFC) showed a significant association between reaction times and activity to negative versus neutral distractors $(p s>.1)$.

\section{DISCUSSION}

Here, we report an effect of COMT val ${ }^{158}$ met genotype on activation in neural regions associated with controlled processing (VLPFC and OFC) and the processing of taskrelated stimuli (bilateral PPA) during performance of a matching task requiring attentional regulation over negative stimuli. Specifically, during conditions requiring the matching of pairs of houses in the presence of negative versus neutral picture distractors, val allele load was positively associated with activation in left VLPFC, left OFC, and bilateral PPA.

The main prediction we were testing was that met allele load would show an inverse correlation with frontal cortical recruitment in response to negative distractors. The effect of COMT genotype on the left VLPFC and left OFC response to house-matching trials with negative versus neutral distractors confirmed this prediction. Bishop, Duncan, Brett, and Lawrence (2004) previously showed that, in the presence of negative distractors, activity in the same left VLPFC region was reduced in high-anxious individuals. In both cases, we would argue that the reduced frontal activation observed may reflect a weakened recruitment of control processes in response to distraction from taskirrelevant negative stimuli. Activation in the left OFC region reported here was not modulated by anxiety in the previous study (Bishop, Duncan, Brett, \& Lawrence, 2004). However, this is possibly a consequence of the fact that the Bruker scanner used in that study gave much poorer coverage of orbital regions with large signal loss in this part of the brain.

Reduced recruitment of controlled processing may impact negatively on the extent to which attentional focus is successfully maintained on task-relevant stimuli. Although there were no differences in behavioral performance associated with COMT genotype, met allele load was also associated with reduced activation during negative distractor trials in the region of parahippocampal cortex known to respond to houses. Given that the task at hand required matching house stimuli and that attentional focus has previously been shown to modulate the PPA response to houses (Bishop, Duncan, \& Lawrence, 2004; Vuilleumier et al., 2001), this is consistent with the proposal that COMT met allele load is associated with weakened attentional focus on task-relevant stimuli in the presence of negative distractors. In keeping with this, there was a trend toward an association between reduced PPA activation to negative (vs. neutral) distractor trials and increased slowing of responses by negative distractors.

The findings reported here hence suggest that the COMT met allele, through its influence on PFC catecholamine metabolism, may reduce the potential for top-down 
regulation of distraction from negative stimuli. This could conceivably operate via a mechanism similar to that underlying the parallel effects of self-reported anxiety (Bishop, Duncan, Brett, \& Lawrence, 2004). It is still too early to make strong suggestions as to how this mechanism may work. However, it is of note that studies of the effects of COMT inhibition in rats indicate a greater effect on PFC catecholamine levels in response to evoked catecholamine release than on baseline PFC catecholamine levels (Tunbridge, Bannerman, Sharp, \& Harrison, 2004). In line with this finding, studies of anxiety disorders have suggested that dysregulation of catecholamine systems in clinically anxious individuals is observed most clearly in response to pharmacological challenge or presentation of negatively valenced stimuli (Southwick et al., 1999). Furthermore, the stress literature suggests that the detrimental effect of stressors on prefrontal function operates via evoked changes in catecholamine release (Arnsten, 1998). Given that the COMT met ${ }^{158}$ allele is associated with decreased catecholamine metabolism, individuals carrying one or two met alleles may show slower baseline recovery in response to catecholamine release triggered by presentation of negative stimuli. Arguably, this might be particularly problematic when these stimuli are not task relevant and when subcortical mechanisms that enable bottom-up, salience-driven stimulus-response processes must be overridden by frontal regulatory mechanisms in order for task focus to be maintained.

The potential importance of the task relevance of negative stimuli is suggested both by previous findings within the attention-emotion literature (see Pessoa, 2005, for a review) and by recent findings by Smolka et al. (2005), who reported a positive association between met allele load and activation in both the limbic system and the VLPFC during passive viewing of negative versus neutral IAPS pictures. This pattern of results is in the opposite direction to that reported here and suggests that the present findings are not driven purely by genotype differences in the response to the presence of negative stimuli in the visual field per se, but that the task being performed is crucial. Indeed, Smolka et al. speculate that the ability of COMT met ${ }^{158}$ allele carriers to recruit prefrontal regulatory regions may be prone to failure under demanding conditions.

As for the possibilities for future work, functional connectivity analyses may throw light on the extent to which activity in the regions more robustly recruited by val allele carriers in response to negative distractors is yoked across time and across different conditions of interest. In the present study, a basic correlational analysis (averaging across the time series for each event type) indicated a substantial extent of covariation between regions associated with controlled processing (VLPFC and OFC) and those associated with task-related processing (PPA), as well as a relationship between OFC and amygdala activity during trials with negative distractors potentially reflecting inhibitory connections (Kim et al., 2004; Logothetis, 2003). More advanced functional connectivity analyses have been used successfully in two recent studies of 5HTT-LPR genotype effects on prefrontal and limbic activation in affective processing paradigms (Heinz et al., 2005; Pezawas et al., 2005). It is of note that the findings of these studies suggest that the connections between prefrontal and cingulate regulatory regions and limbic regions may vary as a function of task and may be differentially modulated by the same genetic marker (in this case, the 5HTT-LPR polymorphism). These results also highlight the importance of considering other common genetic polymorphisms that may have interactive effects with the one under focus. The present study was not of an appropriate scale to investigate COMT val ${ }^{158}$ met $\times$ 5HTT-LPR interactions, but clearly this is an important next step.

As was mentioned earlier, failures to replicate the finding of association studies (linking variants of single genetic polymorphisms to specific psychiatric diagnoses) has led to an emphasis on polygenic models of common psychiatric disorders. For example, whereas some studies have reported a link between the COMT met allele and increased predisposition to clinical anxiety and heightened subclinical anxiety levels, others have failed to do so (Azzam \& Mathews, 2003; Ohara, Nagai, Suzuki, Ochiai, \& Ohara, 1998). This is likely to reflect the complexity of the mechanisms and associated genetic influences involved. We have argued elsewhere that attentional capture by negative or threat-related stimuli may be influenced by an interplay between frontal regulatory mechanisms and subcortical mechanisms that act to prioritize the processing of emotionally salient visual stimuli (Bishop, Duncan, Brett, \& Lawrence, 2004; Bishop, Duncan, \& Lawrence, 2004). As was noted earlier, Hariri et al. (2005; Hariri et al., 2002) have shown that genetic variability in serotonergic mechanisms (e.g., as a result of the 5HTT-LPR polymorphism) may modulate the amygdala response to threat-related stimuli. The strength of this response may well interact with the extent to which PFC control mechanisms promote task-related processing in determining the extent to which an individual is able to avoid unwanted distraction from negative stimuli. Hence, both the 5HTTLPR polymorphism and the COMT val ${ }^{158}$ met polymorphism might influence an individual's ability to regulate the processing of negative distractors, though potentially through distinct neural mechanisms. Furthermore, it is extremely unlikely that these two polymorphisms will be alone in modulating the function of these mechanisms.

We are still at an early stage in establishing the effect of common genetic polymorphisms on neural and cognitive function. Further studies will need to examine gene $x$ gene interactions in the context of neurocognitive models of cognition, emotion, and their interactions. With regard to attentional control over emotional stimuli, this means mapping out genetic effects on both cortical regulatory processes and the processes that determine individual differences in responsiveness to emotional stimuli. Although this is a daunting task, the study reported here is a first step toward analyzing the complex genetic influences that 
underlie both normal variation in affective processing and susceptibility to affective disorders.

\section{REFERENCES}

ARnsten, A. F. T. (1998). Catecholamine modulation of prefrontal cortical cognitive function. Trends in Cognitive Sciences, 2, 436-447.

Azzam, A., \& MATHEws, C. A. (2003). Meta-analysis of the association between the catecholamine-O-methyl-transferase gene and obsessivecompulsive disorder. American Journal of Medical Genetics, 123B, 64-69.

Bilder, R. M., Volavka, J., Lachman, H. M., \& Grace, A. A. (2004). The catechol- $O$-methyltransferase polymorphism: Relations to the tonic-phasic dopamine hypothesis and neuropsychiatric phenotypes. Neuropsychopharmacology, 29, 1943-1961.

Bishop, S. [J.], Duncan, J., Brett, M., \& Lawrence, A. D. (2004). Prefrontal cortical function and anxiety: Controlling attention to threat-related stimuli. Nature Neuroscience, 7, 184-188.

Bishop, S. J., Duncan, J., \& Lawrence, A. D. (2004). State anxiety modulation of the amygdala response to unattended threat-related stimuli. Journal of Neuroscience, 24, 10364-10368.

Brett, M., Anton, J. L., Valabregue, R., \& Poline, J. B. (2002). Region of interest analysis using an SPM toolbox. NeuroImage, 16, 2.

Brown, S. M., \& Hariri, A. R. (2006). Neuroimaging studies of serotonin gene polymorphisms: Exploring the interplay of genes, brain, and behavior. Cognitive, Affective, \& Behavioral Neuroscience, 6, 44-52.

Bruder, G. E., KeIlp, J. G., Xu, H., Shikhman, M., Schori, E., GorMAN, J. M., ET AL. (2005). Catechol- $O$-methyltransferase (COMT) genotypes and working memory: Associations with differing cognitive operations. Biological Psychiatry, 58, 901-907.

Canli, T., Silvers, H., Whitfield, S. L., Gotlib, I. H., \& Gabrieli, J. D. (2002). Amygdala response to happy faces as a function of extraversion. Science, 296, 2191.

Canli, T., Zhao, Z., Desmond, J. E., Kang, E., Gross, J., \& Gabrieli, J. D. (2001). An fMRI study of personality influences on brain reactivity to emotional stimuli. Behavioral Neuroscience, 115, 33-42.

CASEY, B. J. (2005). Frontostriatal and frontocerebellar circuitry underlying cognitive control. In U. Mayr, E. Awh, \& S. W. Keele (Eds.), Developing individuality in the human brain: A tribute to Michael I. Posner (pp. 141-166). Washington, DC: American Psychological Association.

Casey, B. J., Tottenham, N., \& Fossella, J. (2002). Clinical, imaging, lesion, and genetic approaches toward a model of cognitive control. Developmental Psychobiology, 40, 237-254.

Comings, D. E. (2001). Clinical and molecular genetics of ADHD and Tourette syndrome: Two related polygenic disorders. In J. Wasserstein, L. E. Wolf, \& F. F. LeFever (Eds.), Adult attention deficit disorder: Brain mechanisms and life outcomes (Annals of the New York Academy of Sciences, Vol. 931, pp. 50-83). New York: New York Academy of Sciences.

Daniels, J. K., Williams, N. M., Williams, J., Jones, L. A., Cardno, A. G., MurPhy, K. C., ET AL. (1996). No evidence for allelic association between schizophrenia and a polymorphism determining high or low catechol $O$-methyltransferase activity. American Journal of Psychiatry, 153, 268-270.

Duncan, J., \& Owen, A. M. (2000). Common regions of the human frontal lobe recruited by diverse cognitive demands. Trends in Neurosciences, 23, 475-483.

Egan, M. F., Goldberg, T. E., Kolachana, B. S., Callicott, J. H., Mazzanti, C. M., Straub, R. E., ET Al. (2001). Effect of COMT Val108/158 Met genotype on frontal lobe function and risk for schizophrenia. Proceedings of the National Academy of Sciences, 98, 6917-6922.

Enoch, M. A., Xu, K., Ferro, E., Harris, C. R., \& Goldman, D. (2003). Genetic origins of anxiety in women: A role for a functional catechol- $O$ methyltransferase polymorphism. Psychiatric Genetics, 13, 33-41.

EPSTEIN, R., \& KANWISHER, N. (1998). A cortical representation of the local visual environment. Nature, 392, 598-601.

Etkin, A., Klemenhagen, K. C., Dudman, J. T., Rogan, M. T.,
Hen, R., Kandel, E. R., \& Hirsch, J. (2004). Individual differences in trait anxiety predict the response of the basolateral amygdala to unconsciously processed fearful faces. Neuron, 44, 1043-1055.

Fossella, J., Sommer, T., Fan, J., Wu, Y., Swanson, J. M., Pfaff, D. W., \& Posner, M. I. (2002). Assessing the molecular genetics of attention networks. BMC Neuroscience, $3,14$.

Frith, C., \& Dolan, R. J. (1998). Images of psychopathology. Current Opinion in Neurobiology, 8, 259-262.

Goldberg, T. E., Egan, M. F., Gscheidle, T., Coppola, R., WeickERT, T., Kolachana, B. S., et AL. (2003). Executive subprocesses in working memory: Relationship to catechol- $O$-methyltransferase Val158Met genotype and schizophrenia. Archives of General Psychiatry, 60, 889-896.

Goldberg, T. E., \& Weinberger, D. R. (2004). Genes and the parsing of cognitive processes. Trends in Cognitive Sciences, 8, 325-335.

Goldman-RaKic, P. S. (1998). The cortical dopamine system: Role in memory and cognition. Advances in Pharmacology, 42, 707-711.

Gray, J. R., Chabris, C. F., \& Braver, T. S. (2003). Neural mechanisms of general fluid intelligence. Nature Neuroscience, 6, 316-322.

Hariri, A. R., Drabant, E. M., Munoz, K. E., Kolachana, B. S., Mattay, V. S., Egan, M. F., \& Weinberger, D. R. (2005). A susceptibility gene for affective disorders and the response of the human amygdala. Archives of General Psychiatry, 62, 146-152.

Hariri, A. R., Mattay, V. S., Tessitore, A., Kolachana, B., Fera, F., GoldMAN, D., ET AL. (2002). Serotonin transporter genetic variation and the response of the human amygdala. Science, 297, 400-403.

Heinz, A., Braus, D. F., Smolka, M. N., Wrase, J., Puls, I., HermanN, D., ET AL. (2005). Amygdala-prefrontal coupling depends on a genetic variation of the serotonin transporter. Nature Neuroscience, $\mathbf{8}, 20-21$.

Karayiorgou, M., Altemus, M., Galke, B. L., Goldman, D., MurPHY, D. L., Отт, J., \& GoGos, J. A. (1997). Genotype determining low catechol- $O$-methyltransferase activity as a risk factor for obsessivecompulsive disorder. Proceedings of the National Academy of Sciences, 94, 4572-4575.

Kim, H., Somerville, L. H., Johnstone, T., Polis, S., Alexander, A. L., Shin, L. M., \& Whalen, P. J. (2004). Contextual modulation of amygdala responsivity to surprised faces. Journal of Cognitive Neuroscience, 16, 1730-1745.

Lachman, H. M., Morrow, B., Shprintzen, R., Veit, S., Parsia, S. S., FAEDDA, G., ET AL. (1996). Association of codon 108/158 catechol-Omethyltransferase gene polymorphism with the psychiatric manifestations of velo-cardio-facial syndrome. American Journal of Medical Genetics, 67, 468-472.

LanG, P. J., OHMAN, A., \& VaitL, D. (1988). The International Affective Picture System. Gainesville: University of Florida Press.

Lewis, D. A., Sesack, S. R., Levey, A. I., \& Rosenberg, D. R. (1998). Dopamine axons in primate prefrontal cortex: Specificity of distribution, synaptic targets, and development. Advances in Pharmacology, 42, 703-706.

LogOTHETIS, N. K. (2003). The underpinnings of the BOLD functional magnetic resonance imaging signal. Journal of Neuroscience, $\mathbf{2 3}$, 3963-3971.

lotta, T., Vidgren, J., Tilgmann, C., Ulmanen, I., Melen, K., JulKunEN, I., \& TASKINEN, J. (1995). Kinetics of human soluble and membrane-bound catechol $O$-methyltransferase: A revised mechanism and description of the thermolabile variant of the enzyme. Biochemistry, 34, 4202-4210.

Malhotra, A. K., Kestler, L. J., Mazzanti, C., Bates, J. A., GoldBERG, T., \& Goldman, D. (2002). A functional polymorphism in the COMT gene and performance on a test of prefrontal cognition. American Journal of Psychiatry, 159, 652-654.

Mattay, V. S., GoldberG, T. E., Fera, F., Hariri, A. R., Tessitore, A., EgAN, M. F., ET AL. (2003). Catechol $O$-methyltransferase val158met genotype and individual variation in the brain response to amphetamine. Proceedings of the National Academy of Sciences, 100, 6186-6191.

Miller, E. K., \& CohEn, J. D. (2001). An integrative theory of prefrontal cortex function. Annual Review of Neuroscience, 24, 167-202. 
Nolan, K. A., Bilder, R. M., Lachman, H. M., \& Volavka, J. (2004). Catechol O methyltransferase val(158)met polymorphism in schizophrenia: Differential effects of val and met alleles on cognitive stability and flexibility. American Journal of Psychiatry, 161, 359-361.

OChSNER, K. N., \& Gross, J. J. (2005). The cognitive control of emotion. Trends in Cognitive Sciences, 9, 242-249.

Ohara, K., Nagai, M., Suzuki, Y., Ochiai, M., \& Ohara, K. (1998). No association between anxiety disorders and catechol- $O$-methyltransferase polymorphism. Psychiatry Research, 80, 145-148.

Olsson, C. A., Anney, R. J., Lotfi-Miri, M., Byrnes, G. B., Williamson, R., \& Patton, G. C. (2005). Association between the COMT Val158Met polymorphism and propensity to anxiety in an Australian population-based longitudinal study of adolescent health. Psychiatric Genetics, 15, 109-115.

Owen, A. M. (1997). The functional organization of working memory processes within human lateral frontal cortex: The contribution of functional neuroimaging. European Journal of Neuroscience, 9, 1329-1339.

PeSSOA, L. (2005). To what extent are emotional visual stimuli processed without attention and awareness? Current Opinion in Neurobiology, 15, 188-196.

Pezawas, L., Meyer-Lindenberg, A., Drabant, E. M., Verchinski, B. A., Munoz, K. E., Kolachana, B. S., et AL. (2005). 5-HTTLPR polymorphism impacts human cingulate-amygdala interactions: A genetic susceptibility mechanism for depression. Nature Neuroscience, $\mathbf{8}, 828-834$.

Posner, M. I., \& Raichle, M. E. (1995). Precis of images of mind. Behavioral \& Brain Sciences, 18, 327-383.

Sesack, S. R., Hawrylak, V. A., Matus, C., Guido, M. A., \& Levey, A. I. (1998). Dopamine axon varicosities in the prelimbic division of the rat prefrontal cortex exhibit sparse immunoreactivity for the dopamine transporter. Journal of Neuroscience, 18, 2697-2708.

Smolka, M. N., Schumann, G., Wrase, J., Grusser, S. M., Flor, H., MANN, K., ET AL. (2005). Catechol- $O$-methyltransferase val158met genotype affects processing of emotional stimuli in the amygdala and prefrontal cortex. Journal of Neuroscience, 25, 836-842.

Southwick, S. M., Bremner, J. D., Rasmusson, A., Morgan, C. A., III, Arnsten, A., \& Charney, D. S. (1999). Role of norepinephrine in the pathophysiology and treatment of posttraumatic stress disorder. Biological Psychiatry, 46, 1192-1204.

Tunbridge, E. M., Bannerman, D. M., Sharp, T., \& Harrison, P. J. (2004). Catechol- $O$-methyltransferase inhibition improves set-shifting performance and elevates stimulated dopamine release in the rat prefrontal cortex. Journal of Neuroscience, 24, 5331-5335.

Tzourio-Mazoyer, N., Landeau, B., Papathanassiou, D., Crivello, F., Etard, O., Delcroix, N., Et Al. (2002). Automated anatomical labeling of activations in SPM using a macroscopic anatomical parcellation of the MNI MRI single subject brain. NeuroImage, 15, 273-289.

Vuilleumier, P., Armony, J. L., Driver, J., \& Dolan, R. J. (2001). Effects of attention and emotion on face processing in the human brain: An event-related fMRI study. Neuron, 30, 829-841.

Wager, T. D., Rilling, J. K., Smith, E. E., Sokolik, A., Casey, K. L., DAVIDSON, R. J., ET AL. (2004). Placebo-induced changes in FMRI in the anticipation and experience of pain. Science, 303, 1162-1167.

Weinshilboum, R. M., OtTerness, D. M., \& Szumlanski, C. L. (1999). Methylation pharmacogenetics: Catechol $O$-methyltransferase, thiopurine methyltransferase, and histamine $N$-methyltransferase. Аnnual Review of Pharmacology \& Toxicology, 39, 19-52.

Woo, J. M., Yoon, K. S., CHOI, Y. H., OH, K. S., LeE, Y. S., \& Yu, B. H. (2004). The association between panic disorder and the L/L genotype of catechol-O-methyltransferase. Journal of Psychiatric Research, 38, 365-370.

Worsley, K. J., Marrett, S., Neelin, P., Vandal, A. C., Friston, K. J., \& Evans, A. C. (1996). A unified statistical approach for determining significant signals in images of cerebral activation. Human Brain Mapping, 4, 58-73.

Zubieta, J. K., Heitzeg, M. M., Smith, Y. R., Bueller, J. A., Xu, K., XU, Y., ET AL. (2003). COMT val158met genotype affects mu-opioid neurotransmitter responses to a pain stressor. Science, 299, 1240-1243.

\section{NOTES}

1. IAPS pictures replaced the fearful and neutral faces used as negative and neutral distractors in the previous study.

2. Reanalysis with 8-mm-radius spheres did not alter the detection of the $\mathrm{OFC}$ activations reported below.

3. Use of an ANOVA with a $1 / 0 /-1$ ( val val/val met/met met) contrast in place of the simple regression described here produced results equivalent to those reported. The authors thank an anonymous reviewer for this suggestion.

4. PPA activation was extracted from the voxel showing the peak response for the interaction of COMT genotype by distractor valence, as reported above. 